\title{
Percival Variational Principle for Invariant Measures and Commensurate-Incommensurate Phase Transitions in One-Dimensional Chains
}

\author{
V. F. Lazutkin and D. Ya. Terman \\ Research Institute of Physics, Leningrad State University, SU-198904 Leningrad, USSR
}

\begin{abstract}
We prove for a one-dimensional system of classical particles with potential energy,

$$
U_{\alpha, \gamma}=\sum_{n}\left[\alpha V\left(x_{n}\right)+F\left(x_{n+1}-x_{n}-\gamma\right)\right],
$$

the existence of such a smooth function $\gamma(\alpha), 0 \leqq \alpha \leqq \alpha_{0}(\omega)$ that the system with potential energy $U_{\alpha, \gamma(\alpha)}$ has the equilibrium state at the temperature $T=0$. This is the incommensurate phase with the ratio of periods equal to the prescribed irrational number $\omega$, badly approximated by rational ones. A simple geometric condition for the invariant curve of the corresponding dynamical system is established under which it is the support of the invariant measure minimizing Percival's energy functional.
\end{abstract}

\section{Introduction}

The main result of the paper contains the solution of the problem stated in $[1,2]$ and concerns commensurate-incommensurate phase transitions in onedimensional chains. The potential energy for the system has the form

$$
U_{\alpha, \gamma}=\sum_{n}\left[\alpha V\left(x_{n}\right)+F\left(x_{n+1}-x_{n}-\gamma\right)\right] .
$$

Here $x_{n}$ are the coordinates of particles, $V(x)$ is a periodic function with period 1 having nondegenerate minima at $x=n$ and maxima at $x=n+\frac{1}{2}, n \in \mathbb{Z}, F$ is the potential energy of the inner interaction between nearest neighbours, and $\alpha$ and $\beta$ are parameters. We assume also that $F$ is strictly convex, $F^{\prime \prime} \geqq$ const $>0, F(0)$ $=F^{\prime}(0)=0$.

As it was shown in [1] the phase diagram of the model for the temperature $T=0$ is described in terms of invariant measures of mappings of the twodimensional cylinder $C=S^{1} \times \mathbb{R}$. The transformation for (1.1) is defined as follows: $f(x, y)=\left(x^{\prime}, y^{\prime}\right)$, where

$$
\begin{aligned}
y & =-\alpha V^{\prime}(x)+F^{\prime}\left(x^{\prime}-x-\gamma\right), \\
y^{\prime} & =F^{\prime}\left(x^{\prime}-x-\gamma\right) .
\end{aligned}
$$


In the case of Frenkel-Kontorova model (see [3]) $F(x)=\frac{1}{2} x^{2}, \quad V(x)$ $=-(2 \pi)^{-1} \cos 2 \pi x$, and the transformation $f$ coincides with the well-known standard or Chirikov's map in the theory of non-linear oscillations (see [4]). For any normed measure $\mu$ on $C$ with compact support and invariant with respect to $f$ we put

$$
P(\mu)=\int\left[\alpha V(x)+F\left(x^{\prime}-x-\gamma\right)\right] d \mu .
$$

Here $x^{\prime}=\pi_{1} f(x, y), \pi_{1}$ is the projection of the cylinder $C=S^{1} \times \mathbb{R}$ to the first component. According to [1] limit points of Gibbs states of the system in question when $T \rightarrow 0$ are the measures on the configuration space concentrating on the space of trajectories of $f$, and corresponding invariant under $f$ measures $\mu$ on $C$ are those for which $P(\mu)$ takes minimal value. A question naturally arises to describe the invariant measures $\mu_{\alpha, \gamma}$ for which $P\left(\mu_{\alpha, \gamma}\right)=\min _{\mu \in \mathfrak{M}_{f}} P(\mu)$, where $\mathfrak{M}_{f}$ is the set of all normed measures invariant with respect to $f$ having compact support. The map $(\alpha, \gamma) \mapsto \mu_{\alpha, \gamma}$ is the phase diagram of the system (this map is not necessarily singlevalued). This question is very delicate and difficult to answer in view of the complexity of the partition of the cylinder $C$ onto invariant sets with respect to $f$ and the presence of stochastic layers (see [4]). Therefore, we reverse the formulation of the problem and ask for any given invariant measure $\bar{\mu}$, whether it is true that $P(\bar{\mu})=\min _{\mu \in \mathfrak{M}_{f}} P(\mu)$. We shall consider the case when the support of $\bar{\mu}$ is contained in some homotopically nontrivial circle $z$ invariant with respect to $f$. Such $\bar{\mu}$ is unique if $f$ is topologically transitive on $z$. In this case $f$ restricted to $z$ can be reduced to a rotation and the minimal property is physically interpreted as the appearance of an incommensurate phase at $T=0$ (see $[1,2,12-14])$. Theorem A stated in Sect. 4 gives a simple geometrical answer to this question: let the invariant circle $z$ be the graph of the periodic function $\psi(x)$ with period 1 . Then the invariant measure $\mu$ with supp $\mu \subset z$ minimizes $P$ if and only if $z$ bounds zero area, i.e. $\int_{0}^{1} \psi(x) d x=0$.

Let us consider a slightly modified question. Fix the irrational number $\omega$, which is badly approximated by rational ones. According to Kolmogorov-ArnoldMoser theory (KAM theory) the transformation $f$ has an invariant homotopically nontrivial circle with the rotation number equal to $\omega$ if $\alpha$ is sufficiently small (see [5]). In this case such a circle $z_{\omega}$ is unique (see [6]) and the invariant measure $\mu_{\omega}$ with support belonging to this circle is also unique. Do the pairs $(\alpha, \gamma)$ exist such that the invariant measure $\mu_{\alpha, \gamma}$ giving the minimum to $P(\mu)$ coincides with $\mu_{\omega}$ ? In this formulation the problem becomes very close to the variational principle for invariant sets of KAM theory investigated recently by Percival [7] and Mather [8]. In the case of the Frenkel-Kontorova model the answer to this question is an immediate consequence of the theorem mentioned above. To see this, write the formulas (1.2) for this case as follows:

$$
x^{\prime}=x+y^{\prime}+\gamma, \quad y^{\prime}=y+\alpha V^{\prime}(x),
$$

and denote the corresponding transformation of $C$ by $f_{\alpha, \gamma}$, indicating explicitly the dependence on $\alpha$ and $\gamma$. If $f_{\alpha, 0}$ has the invariant circle $z_{\omega}$ with the rotation number $\omega$ 
and $\jmath_{\omega}=\operatorname{graph} \psi$, then $f_{\alpha, \gamma}$ has the invariant circle with the same rotation number which is the graph of function $\psi-\gamma$. So the value of $\gamma$ for which the invariant circle $3_{\omega}$ bounds the zero area is $\gamma=\int_{0}^{1} \psi(x) d x$. Using KAM theory we have for the Frenkel-Kontorova model the existence of a smooth function $\gamma(\alpha), 0 \leqq \alpha \leqq \alpha_{0}(\omega)$, such that the incommensurate phase with the ratio of periods equal to $\omega$ exists for any pair $(\alpha, \gamma(\alpha)), 0 \leqq \alpha \leqq \alpha_{0}(\omega)$. This is true when $F(y)$ is a quadratic function. In the more general case one needs some more complicated considerations to establish the existence of such $\gamma(\alpha)$ (see Sect. 8). Another approach to the commensurateincommensurate phase transitions is developed in recent papers $[13,14]$.

It is worthwhile to mention a recent result by Zaslavski [9] for the FrenkelKontorova model, where he has shown for $\gamma \neq n+\frac{1}{2}$ that one could find $\alpha_{1}=\alpha_{1}(\gamma)$ such that for $\alpha>\alpha_{1}(\gamma)$ the invariant measure $\mu_{\alpha, \gamma}$ is concentrated at the hyperbolic fixed point $x=0, y=-\gamma$.

\section{Exact Mappings}

In this section we introduce a class of dynamical systems which are useful for describing the equilibrium configurations of one-dimensional chains. It is more convenient to work with the universal cover of the cylinder $C=S^{1} \times \mathbb{R}$, that is with $\mathbb{R}^{2}$. So we consider the translation $T: \mathbb{R}^{2} \rightarrow \mathbb{R}^{2}, T(x, y)=(x+1, y)$, and projections $\pi_{i}: \mathbb{R}^{2} \rightarrow \mathbb{R}, i=1,2$, defined by $\pi_{1}(x, y)=x, \pi_{2}(x, y)=y$. Let $f: \mathbb{R}^{2} \rightarrow \mathbb{R}^{2}$ be a $C^{1}$-mapping and put

$$
\begin{gathered}
d f(x, y)=\left(\begin{array}{ll}
\frac{\partial \pi_{1} f(x, y)}{\partial x} & \frac{\partial \pi_{1} f(x, y)}{\partial y} \\
\frac{\partial \pi_{2} f(x, y)}{\partial x} & \frac{\partial \pi_{2} f(x, y)}{\partial y}
\end{array}\right), \\
\|d f\|=\sup _{(x, y) \in \mathbb{R}^{2}}\|d f(x, y)\|,
\end{gathered}
$$

where the norm of $2 \times 2$ matrix $\mathscr{A}=\left\{a_{i k}\right\}_{i, k=1}^{2}$ is defined by the expression $\|\mathscr{A}\|=\max _{i, k}\left|a_{i k}\right|$.

Let $\mathscr{D}$ denote the set of all diffeomorphisms $f: \mathbb{R}^{2} \rightarrow \mathbb{R}^{2}$ belonging to the class $C^{1}$, commuting with $T$, and having the uniformly bounded first derivatives, i.e. $f \in \mathscr{D} \stackrel{\text { def }}{\Leftrightarrow}(\mathscr{D} 1) \wedge(\mathscr{D} 2) \wedge(\mathscr{D} 3)$, where

(ஜ1) $f: \mathbb{R}^{2} \rightarrow \mathbb{R}^{2}$ is a $C^{1}$-diffeomorphism;

(D2) $f \circ T=T \circ f$;

(ஜ)3) $\|d f\|<\infty$.

Definition. The map $f \in \mathscr{D}$ is called exact if there exists a $C^{2}$-function $L: \mathbb{R}^{2} \rightarrow \mathbb{R}$ having the properties (L1), (L2), and (L3):

(L1) $L\left(x, x^{\prime}\right)=L\left(x+1, x^{\prime}+1\right)$, 
(L2) $\sup _{(x, x) \in \mathbb{R}^{2}}\left|\frac{\partial^{2} L\left(x, x^{\prime}\right)}{\partial x \partial x^{\prime}}\right|<\infty$,
(L3) $\left(x^{\prime}, y^{\prime}\right)=f(x, y) \Leftrightarrow y=-\frac{\partial L\left(x, x^{\prime}\right)}{\partial x}, y^{\prime}=\frac{\partial L\left(x, x^{\prime}\right)}{\partial x^{\prime}}$.

A function $L$ will be called a generating function for the map $f$ and will be denoted by $L_{f}$ if it is needed to indicate the dependence on $f$. The set of all exact mappings will be denoted by $\mathscr{E}$.

Remark. Two generating functions for the same $f \in \mathscr{E}$ differ only by a constant.

Denote by 3 the set of all curves in $\mathbb{R}^{2}$, invariant with respect to the translation $T$, which are the graphs of $C^{2}$-functions, i.e. $z \in \mathcal{B} \Leftrightarrow\left[\exists \psi \in C^{1}(\mathbb{R}, \mathbb{R}), \psi(x+1)\right.$ $=\psi(x), \forall x \in \mathbb{R}$, and $\left.(x, y) \in \mathfrak{z} \Leftrightarrow y=\psi(x), \forall(x, y) \in \mathbb{R}^{2}\right]$. Define the mapping $\beta: 3 \rightarrow \mathbb{R}$ by the formula

$$
\beta(z)=\int_{0}^{1} \psi(x) d x, \quad z=\operatorname{graph} \psi .
$$

The next proposition characterizes exact mappings.

Proposition 2.1. Let $f \in \mathscr{D}$. Then

$$
f \in \mathscr{E} \Leftrightarrow I(f) \wedge I I(f) \wedge I I I(f),
$$

where

$$
\begin{aligned}
I(f) & \Leftrightarrow f \text { preserves area and orientation, } \\
I I(f) & \Leftrightarrow \inf \left|\frac{\partial \pi_{1} f(x, y)}{\partial y}\right|>0 \text { ("twist condition"), } \\
I I I(f) & \Leftrightarrow \beta(f(\mathfrak{z}))=\beta(\jmath), \forall z \in 3 .
\end{aligned}
$$

Proposition 2.1 will be proven in Sect. 9.

Corollary 2.2. $\mathscr{E}=\mathscr{E}_{+} \cup \mathscr{E}_{-}, \mathscr{E}_{+} \cap \mathscr{E}_{-}=\emptyset$, where $\mathscr{E}_{ \pm}$is the set of all $f \in \mathscr{E}$ for which sign $\frac{\partial \pi_{1} f(x, y)}{\partial y}= \pm 1$ (positive and negative twist condition).

\section{Measures}

Let $\mathscr{C}$ be the space of all continuous functions $\varphi: \mathbb{R}^{2} \rightarrow \mathbb{R}$, invariant with respect to $T(\varphi \circ T=\varphi)$, with the structure of real vector space defined in the usual manner; $\operatorname{supp} \varphi$ for $\varphi \in \mathscr{C}$ will denote the support of $\varphi$, i.e. the closure of the set of points $(x, y) \in \mathbb{R}^{2}$ for which $\varphi(x, y) \neq 0$. For $b>0$ we put $A_{b}=\left\{(x, y) \in \mathbb{R}^{2}:|y| \leqq b\right\}$ (the strip of the halfwidth $b$ ).

Definition. A measure is a map $\mu: \mathscr{C} \rightarrow \mathbb{R}$ which is 1) linear, 2) nonnegative: $\varphi \geqq 0 \Rightarrow \mu(\varphi) \geqq 0, \forall \varphi \in \mathscr{C}, 3)$ normed: $\mu(1)=1$, and 4) having bounded support in the $y$-direction: $\exists b>0, \forall \varphi \in \mathscr{C} \operatorname{supp} \varphi \cap A_{b}=\emptyset \Rightarrow \mu(\varphi)=0$. We denote the set of all such measures by $\mathfrak{M}$. 
Definition. Let $\mu \in \mathfrak{M}$. The support of measure $\mu$ is the set $\operatorname{supp} \mu \subset \mathbb{R}^{2}$, which is defined by the condition: $(x, y) \& \operatorname{supp} \mu \Leftrightarrow \exists$ open set $\mathscr{G} \subset \mathbb{R}^{2}$ such that $(x, y) \in \mathscr{G}$ and $\forall \varphi \in \mathscr{C} \operatorname{supp} \varphi \subset \mathscr{G} \Rightarrow \mu(\varphi)=0$.

The support of a measure is the closed set invariant with respect to $T$ which is contained in a strip $A_{b}$. For $\varphi \in \mathscr{C}$, let $N(\varphi)$ denote the set of zeroes of the function $\varphi$. The following proposition is obvious.

Proposition 3.1. Let $\varphi \in \mathscr{C}, \varphi \geqq 0, \mu \in \mathfrak{M}$. Then

$$
\mu(\varphi)=0 \Leftrightarrow \operatorname{supp} \mu \subset N(\varphi) .
$$

Remark. $\mathfrak{M}$ is a convex subset of the space algebraically conjugated to $\mathscr{C}$.

We shall define for $f \in \mathscr{D}$ a linear map $f^{*}: \mathscr{C} \rightarrow \mathscr{C}$ by the formula $f^{*} \varphi=\varphi \circ f$, and affine map $f_{*}: \mathfrak{M} \rightarrow \mathfrak{M}$ by the formula $f_{*} \mu=\mu \circ f^{*}$. The maps $f^{*}$ and $f_{*}$ are bijections with the following properties: $(f \circ g)^{*}=g^{*} \circ f^{*},(f \circ g)_{*}=f_{*} \circ g_{*}, \operatorname{supp} f_{*} \mu$ $=f(\operatorname{supp} \mu)$. The set of all measures invariant with respect to $f$ will be denoted by $\mathfrak{M}_{f}$, it is a convex subset of $\mathfrak{M}$. One could easily verify for any $f, g \in \mathscr{D}$

$$
g_{*}\left(\mathfrak{M}_{f}\right)=\mathfrak{M}_{g \circ f \circ g^{-1}}
$$

\section{Variational Principle}

Let $f \in \mathscr{E}$ and $L_{f}$ be a generating function for $f$. We define Percival's functional $P_{f}: \mathfrak{M}_{f} \rightarrow \mathbb{R}$ by the formula

$$
P_{f}(\mu)=\int L_{f}\left(x, x^{\prime}\right) d \mu \stackrel{\text { def }}{=} \mu(F),
$$

where

$$
F(x, y)=L_{f}\left(x, \pi_{1} f(x, y)\right) .
$$

The curve $z \in 3$ will be called invariant with respect to $f$ if $f(z) \subset z$. An invariant curve is said to be a zero curve if $\beta(3)=0$ [see (2.3) for the definition of $\beta(3)$ ].

Theorem A. Let the mapping $f \in \mathscr{E}_{+}$have an invariant zero curve $z \in 3$. Then the following statements are valid for any measure $\left.\mu \in \mathfrak{M}_{f}: 1\right)$ If $\operatorname{supp} \mu \mathrm{C}_{3}$, then $P_{f}(\mu)=P_{0}$, where $P_{0}$ is the same for all such measures. 2) If supp $\mu \not \subset z$, then $P_{f}(\mu)>P_{0}$.

Remark 4.1. If $f \in \mathscr{E}_{-}$the statements remain valid if one replaces sign $>$by $<$in 2 ).

Remark 4.2. If the curve $z$ is not a zero one, i.e. $\beta=\beta(\mathfrak{z}) \neq 0$, then the statements of the theorem will be valid if one replaces function $L_{f}\left(x, x^{\prime}\right)$ in (4.1) by $L_{f}\left(x, x^{\prime}\right)+\beta$ $\cdot\left(x-x^{\prime}\right)$.

Theorem A and Remark 4.1 will be proven in Sect. 7, Remark 4.2 in Sect. 11.

\section{Invariance of Percival's Functional with Respect to Exact Transformations}

Proposition 5.1. Let $f, g \in \mathscr{E}$, and $\tilde{f}=g \circ f \circ g^{-1}$ satisfies the twist condition $I I(\tilde{f})$ of Proposition 2.1. Then $\tilde{f} \in \mathscr{E}$ and $P_{f}=P_{\tilde{f}} \circ g_{*}+$ const.

This proposition will be proven in Sect. 9. 


\section{Straightening Transformations}

Let $f \in \mathscr{E}$ and $z=\operatorname{graph} \psi \in 3$ be a zero curve invariant with respect to $f$. A straightening transformation $g: \mathbb{R}^{2} \rightarrow \mathbb{R}^{2}$ for the pair $(f, \precsim)$ is defined by the formula $g(x, y)=(\tilde{x}, \tilde{y})$, where

$$
\tilde{x}=x+\varepsilon \tilde{y}, \quad \tilde{y}=y-\psi(x) .
$$

This transformation is exact if $\varepsilon \neq 0$. The generating function of $g$ has the form:

$$
L_{g}(x, \tilde{x})=-\int_{0}^{x} \psi(\tau) d \tau+\frac{1}{2 \varepsilon}(x-\tilde{x})^{2} .
$$

Note that $\beta(3)=0$ is equivalent to the condition (L1) for $L_{g}$.

Proposition 6.1. Let $g$ be a straightening transformation for the pair $(f, \mathfrak{\jmath})$. If $\varepsilon$ is sufficiently small then $\tilde{f}=g \circ f \circ g^{-1}$ satisfies the twist condition II $(\widetilde{f})$ of Proposition 2.1.

Proposition 6.1 will be proven in Sect. 10.

Proposition 5.1 shows that in this case $\tilde{f} \in \mathscr{E}$ and $P_{f}=P_{\widetilde{f}^{\circ}} g_{*}+$ const. Moreover, the straightening transformation transforms the invariant curve $z$ into the $x$-axis. So it is sufficient to prove Theorem A for the case when $z$ is the $x$-axis.

\section{Proof of Theorem A}

We may assume that $f \in \mathscr{E}_{+}$and

$$
\pi_{2} f(x, 0)=0 \text { for all } x \in \mathbb{R} .
$$

Let us calculate the derivatives of the function $F(x, y)$ given by (4.2)

$$
\begin{aligned}
\frac{\partial F(x, y)}{\partial x} & =\left.\frac{\partial L\left(x, x^{\prime}\right)}{\partial x}\right|_{x^{\prime}=\pi_{1} f(x, y)}+\left.\frac{\partial L\left(x, x^{\prime}\right)}{\partial x^{\prime}}\right|_{x^{\prime}=\pi_{1} f(x, y)} \cdot \frac{\partial \pi_{1} f(x, y)}{\partial x} \\
& =-y+y^{\prime} \frac{\partial \pi_{1} f(x, y)}{\partial x}, \\
\frac{\partial F(x, y)}{\partial y} & =\left.\frac{\partial L\left(x, x^{\prime}\right)}{\partial x^{\prime}}\right|_{x^{\prime}=\pi_{1} f(x, y)} \cdot \frac{\partial \pi_{1} f(x, y)}{\partial y}=y^{\prime} \frac{\partial \pi_{1} f(x, y)}{\partial y} .
\end{aligned}
$$

In these formulas $y^{\prime}=\pi_{2} f(x, y)$. Since $f$ is area and orientation preserving, the condition (7.1) gives

$$
\left.\frac{\partial \pi_{1} f(x, 0)}{\partial x} \cdot \frac{\partial \pi_{2} f(x, y)}{\partial y}\right|_{y=0}=1
$$

Thus $\frac{\partial \pi_{1} f(x, 0)}{\partial x}$ does not vanish and is positive because of commuting property of

$$
\begin{aligned}
& f: \pi_{1} f(x+1, y)=\pi_{1} f(x, y)+1 . \text { So }\left.\frac{\partial \pi_{2} f(x, y)}{\partial y}\right|_{y=0}>0 \text { and } \\
& y=0 \Leftrightarrow y^{\prime}=0, \\
& y>0 \Leftrightarrow y^{\prime}>0, \\
& y<0 \Leftrightarrow y^{\prime}<0 .
\end{aligned}
$$


Since $f \in \mathscr{E}_{+}$we have

$$
\frac{\partial \pi_{1} f(x, y)}{\partial y}>0
$$

One can easily deduce from (7.2)-(7.5) that the graph of $F$ is channelwise, i.e. $F$ is constant on the $x$-axis and strictly increases if one moves in both $y$-directions. The statement of Theorem A is then the consequence of Proposition 3.1. If $f \in \mathscr{E}_{-}$one should change the sign in (7.5) by the opposite one. So the graph of function $F$ would be humpwise and we make sure of the validity of Remark 4.1.

\section{Existence of an Incommensurate Phase}

Let the Lagrangian $L\left(x, x^{\prime}\right)$ have the form

$$
L\left(x, x^{\prime}\right)=\alpha V(x)+F\left(x^{\prime}-x-\gamma\right),
$$

as we have discussed in Sect. 1. Here $\alpha$ is a small parameter, $F(y)$ and $V(x)$ are $C^{\infty}$-functions defined on the real axis and satisfying the following conditions: $F(0)$ $=F^{\prime}(0)=0, F^{\prime \prime}(y) \geqq$ const $>0, \forall y \in \mathbb{R}, V(x+1)=V(x), \forall x \in \mathbb{R}$. The corresponding transformation $f_{\alpha, \gamma}$ is $f_{\alpha, \gamma}(x, y)=\left(x^{\prime}, y^{\prime}\right)$, where

$$
x^{\prime}=x+\gamma+\left(F^{\prime}\right)^{-1}\left(y^{\prime}\right), \quad y^{\prime}=y+\alpha V^{\prime}(x) \text {. }
$$

As a consequence of Theorem $A$ we have that the unique incommensurate phase with irrational ratio of periods $\omega$ in the one-dimensional chain with potential energy (1.1) exists if the transformation $f_{\alpha, \gamma}$ have the zero invariant curve $z \in 3$ with rotation number $\omega$. We shall prove the existence of such a curve using the KAM theory in a modified form due to Zehnder [10] and Hamilton [11]. To apply the KAM theory we must take $\omega$ satisfying the following arithmetical condition:

$$
|n \omega-m| \geqq c_{0} n^{-\sigma}, \quad \forall n, m \in \mathbb{Z}, \quad n \neq 0,
$$

where $c_{0}>0, \sigma>1$.

Theorem B. Let $\omega$ satisfy the arithmetical condition (8.3). Then there exists a $C^{\infty}$-function $\gamma(\alpha), 0 \leqq \alpha<\alpha_{0}=\alpha_{0}\left(V, F, c_{0}, \sigma\right)$ such that the transformation $f_{\alpha, \gamma(\alpha)}$ has an invariant zero curve $z_{\omega, \alpha} \in 3$ with rotation number $\omega$. Such a curve is unique and $C^{\infty}$-depending on $\alpha: z_{\omega, 0}$ is the $x$-axis, $\gamma(0)=\omega$.

Proof. We shall apply the implicit function theorem with a quadratic error (see [11, Theorem 3.3.1, on p. 211]). So we consider the spaces $\mathscr{F}=C^{\infty}\left(S^{1}, \mathbb{R}^{2}\right) \times \mathbb{R}^{2}$, $\mathscr{G}=\mathbb{R}, \mathscr{H}=C^{\infty}\left(S^{1}, \mathbb{R}^{2}\right) \times \mathbb{R}$, where $S^{1}$ is the circle $S^{1}=\mathbb{R} / \mathbb{Z}$, and the map $A: \mathscr{F} \times \mathscr{G} \rightarrow \mathscr{H}$ defined by formulas $A(\varphi, \alpha)=\left(A_{1}, A_{2}\right), \varphi=(\mathfrak{\jmath}, \gamma, \delta) \in \mathscr{F}, \alpha \in \mathscr{G}$, $A_{1} \in C^{\infty}\left(S^{1}, \mathbb{R}^{2}\right), A_{2} \in \mathbb{R}$,

$$
A_{1}(\varphi, \alpha)(t)=z(t+\omega)-f_{\alpha, \gamma, \delta}(z(t)), \quad A_{2}(\varphi, \alpha)=\beta(\jmath) .
$$

Instead of the transformation $f_{\alpha, \gamma}: \mathbb{R}^{2} \rightarrow \mathbb{R}^{2}$ we shall consider the transformation $f_{\alpha, \gamma, \delta}: S^{1} \times \mathbb{R} \rightarrow S^{1} \times \mathbb{R}$, which is defined by formulas $f_{\alpha, \gamma, \delta}(x, y)=\left(x^{\prime}, y^{\prime}\right)$,

$$
x^{\prime}=x+\gamma+\left(F^{\prime}\right)^{-1}\left(y^{\prime}\right)(\bmod 1), \quad y^{\prime}=y+\delta+\alpha V^{\prime}(x),
$$


introducing an additional parameter $\delta$. The lifting of $f_{\alpha, \gamma, \delta}$ on $\mathbb{R}^{2}$ is exact if and only if $\delta=0$, then it coincides with $f_{\alpha, \gamma}$. Supplying the space in question by appropriate systems of norms we turn them into so-called Frechet tame spaces, and the map $A$ becomes the tame map (see [11]). If we take $\varphi_{0}=(0, \omega, \omega), \alpha=0$, then

$$
A\left(\varphi_{0}, 0\right)=0 \text {. }
$$

One can compute the derivative $D_{\varphi} A(\varphi, \alpha)$ of the map $A$ as follows:

$$
\begin{aligned}
& D_{\varphi} A(\varphi, \alpha) \tilde{\varphi}=\left(D_{\varphi} A_{1}(\varphi, \alpha) \tilde{\varphi}, D_{\varphi} A_{2}(\varphi, \alpha) \tilde{\varphi}\right), \quad \tilde{\varphi}=(\tilde{\jmath}, \tilde{\gamma}, \tilde{\delta}), \\
& D_{\varphi} A_{1}(\varphi, \alpha) \tilde{\varphi}(t)=\tilde{\jmath}(t+\omega)-d f_{\alpha, \gamma, \delta}(\mathfrak{z}(t)) \tilde{z}(t)-\tilde{\gamma} e_{1}-\tilde{\delta} e_{2}, \\
& e_{1}=(1,0), \quad e_{2}=(0,1) \in \mathbb{R}^{2}, \\
& D_{\varphi} A_{2}(\varphi, \alpha) \tilde{\varphi}= \int_{0}^{1}\left[\pi_{2}(t) d \pi_{1} \tilde{\jmath}(t)+\pi_{2} \tilde{\jmath}(t) d \pi_{1}(t)\right] .
\end{aligned}
$$

We take the basis $\{v(t), w(t)\}$ in the tangent space to $\mathbb{R}^{2}$ at the point $z(t)$, putting $v(t)=\frac{d_{3}(t)}{d t}, d x \wedge d y(v(t), w(t))=1$, and the basis $(v(t+\omega), w(t+\omega))$ in the tangent space to $\mathbb{R}^{2}$ at the point $f(z(t))$. Then in these basises the matrix of $d f$ takes the form

$$
\left(\begin{array}{cc}
1 & a(t) \\
0 & 1
\end{array}\right)+\left(\begin{array}{cc}
-A_{11}^{\prime}(t) & 0 \\
-A_{12}^{\prime}(t) & A_{11}^{\prime}(t) b(t)-A_{12}^{\prime}(t) a(t)
\end{array}\right),
$$

where $A_{11}(t)$ and $A_{12}(t)$ are the components of $A_{1}(\varphi, \alpha)(t)$ in the basis $\{v(t+\omega), w(t+\omega)\}$. Let us construct the approximate right inverse operator $\mathbf{V}(\varphi, \alpha): \mathscr{H} \rightarrow \mathscr{F}$, provided $(\varphi, \alpha)$ is sufficiently close to $\left(\varphi_{0}, 0\right)$. For $h=\left(h_{1}, h_{2}\right) \in \mathscr{H}$ $=C^{\infty}\left(S^{1}, \mathbb{R}^{2}\right) \times \mathbb{R}$, we denote by $h_{11}(t)$ and $h_{12}(t)$ the components of $h_{1}(t)$ in the basis $\{v(t+\omega), w(t+\omega)\}$. We shall define the operator $\mathbf{V}(\varphi, \alpha)$ by the formula $\mathbf{V}(\varphi, \alpha) h=(\tilde{\jmath}, \tilde{\gamma}, \tilde{\delta})$, where $\tilde{\jmath} \in C^{\infty}\left(S^{1}, \mathbb{R}^{2}\right), \tilde{\gamma}, \tilde{\delta} \in \mathbb{R}$ are defined as a solution of the following system:

$$
\begin{gathered}
\tilde{z}_{1}(t+\omega)-\tilde{z}_{1}(t)-a(t) \tilde{z}_{2}(t)-a_{11}(t) \tilde{\gamma}-a_{21}(t) \tilde{\delta}=h_{11}(t), \\
\tilde{z}_{2}(t+\omega)-\tilde{z}_{2}(t)-a_{12}(t) \tilde{\gamma}-a_{22}(t) \tilde{\delta}=h_{12}(t), \\
\int_{0}^{1}\left[\pi_{2} z(t) d \pi_{1} \tilde{z}(t)+\pi_{2} \tilde{z}(t) d \pi_{1} z(t)\right]=h_{2}, \\
\int_{0}^{1} \tilde{z}_{1}(t) d t=0, \\
-\int_{0}^{1} a_{12}(t) d t \tilde{\gamma}-\int_{0}^{1} a_{22}(t) d t \tilde{\delta}=\int_{0}^{1} h_{12}(t) d t, \\
-\int_{0}^{1} a(t) \tilde{z}_{2}(t) d t-\int_{0}^{1} a_{11}(t) d t \tilde{\gamma}-\int_{0}^{1} a_{21}(t) d t \tilde{\delta}=\int_{0}^{1} h_{11}(t) d t
\end{gathered}
$$

Here $\tilde{z}_{1}(t)$ and $\tilde{z}_{2}(t)$ are the components of $\tilde{z}(t)$ in the basis $\{v(t), w(t)\} ; a_{i j}(t)$ are the components of $e_{i}$ in the basis $\{v(t+\omega), w(t+\omega)\}$. We shall require the periodicity of $\tilde{z}_{j}(t)$ with period 1 .

The solution of $(8.8)$ is

$$
\tilde{\jmath}_{j}(t)=\sum_{n \neq 0}\left(e^{i n 2 \pi \omega}-1\right)^{-1} \tilde{h}_{j n}+\tilde{z}_{j 0}
$$


where $\tilde{h}_{j n}, j=1,2$, are the Fourier coefficients of the functions $h_{11}(t)+a(t)+a_{11}(t) \tilde{\gamma}$ $+a_{21}(t) \tilde{\delta}, h_{12}(t)+a_{12}(t) \tilde{\gamma}+a_{22}(t) \tilde{\delta}$, respectively, $\tilde{z}_{j 0} \in \mathbb{R}$. Note that (8.9) give us the zero Fourier coefficients $\tilde{h}_{j 0}, j=1,2$. Let us consider (8.9) as the system of equations with respect to real variables $\left(\tilde{z}_{10}, \tilde{z}_{20}, \tilde{\gamma}, \tilde{\delta}\right)$. This system is linear. If $(\varphi, \alpha)$ is sufficiently close to $\left(\varphi_{0}, 0\right)$, then the determinant of the system $(8.9)$ is separated from zero, and $\left(\tilde{z}_{10}, \tilde{z}_{20}, \tilde{\gamma}, \tilde{\delta}\right)$ are smooth functions of $(\varphi, \alpha)$. We can solve the last pair of equations $(8.9)$ separately and substitute $(\tilde{\gamma}, \tilde{\delta})$ into $(8.8)$. Then $\tilde{z}_{j}(t)$ will be defined by (8.10), where $\tilde{z}_{j 0}$ in turn will be defined as the solution of the first pair of (8.9).

We have

$$
D_{\varphi} A(\varphi, \alpha) \mathbf{V}(\varphi, \alpha) h=h+\mathscr{Q}(A, h)
$$

where

$$
\begin{gathered}
\mathscr{2}(k, h)=\left(\mathscr{Q}_{11}, \mathscr{Q}_{12}, 0\right) \in \mathscr{H}, \\
\mathscr{Q}_{11}(t)=k_{11}^{\prime}(t)(\mathbf{V}(\varphi, \alpha) h)_{11}(t), \\
\mathscr{Q}_{12}(t)=k_{12}^{\prime}(t)(\mathbf{V}(\varphi, \alpha) h)_{11}(t)+\left(k_{12}^{\prime}(t) a(t)-k_{11}^{\prime}(t)\right)(\mathbf{V}(\varphi, \alpha))_{12}(t) .
\end{gathered}
$$

Let $U \subset \mathscr{F} \times \mathscr{G}$ be a sufficiently small neighbourhood of $\left(\varphi_{0}, 0\right)$, in which the preceding considerations are valid. The maps $\mathbf{V}: U \times \mathscr{H} \rightarrow \mathscr{F}$ and $\mathscr{Q}: U \times \mathscr{H} \times \mathscr{H} \rightarrow \mathscr{H}$ satisfy all the conditions of Hamilton's theorem 3.3.1 [11], and we can assert the existence of solution $(\jmath, \gamma, \delta)$ of the system $A(\varphi, \alpha)=0$ in some neighbourhood of $\alpha=0$ smoothly dependent on $\alpha$. The curve $z$ will be the invariant zero curve with respect to $f_{\alpha, \gamma, \delta}$ having rotation number $\omega$. Since $f_{\alpha, \gamma, \delta}$ preserves area and has an invariant curve, its lifting is exact, and so $\delta=0$. The uniqueness of the invariant circle with irrational rotation number is proved in [6, Proposition 4.1].

\section{Proof of Propositions 2.1 and 5.1}

Lemma 9.1. Let $f \in \mathscr{D}$, then $I(f) \wedge I I(f) \Leftrightarrow \exists C^{\infty}$-function $L: \mathbb{R}^{2} \rightarrow \mathbb{R}$, satisfying conditions (L2) and (L3) of Sect. 2.

Proof. $\Leftarrow)$ Let $f(x, y)=\left(x^{\prime}, y^{\prime}\right)$. (L3) yields

$$
y^{\prime} d x^{\prime}-y d x=d L\left(x, x^{\prime}\right)
$$

and $d y^{\prime} \wedge d x^{\prime}=d y \wedge d x$, so $I(f)$ is valid. By differentiating the equation $y=-\frac{\partial L\left(x, x^{\prime}\right)}{\partial x}$, one can write

$$
1=-\frac{\partial^{2} L\left(x, x^{\prime}\right)}{\partial x \partial x^{\prime}} \cdot \frac{\partial \pi_{1} f(x, y)}{\partial y}
$$

which yields the equivalency of (L2) and $I I(f)$.

$\Rightarrow$ ) Consider the function $\Phi(y)=\pi_{1} f(x, y)-x^{\prime}$, provided $x$ and $x^{\prime}$ are fixed. Condition $I I(f)$ yields

$$
\left|\frac{d \Phi(y)}{d y}\right| \geqq c_{0}>0,
$$


so $\Phi: \mathbb{R} \rightarrow \mathbb{R}$ is the bijection. Define the function $g: \mathbb{R}^{2} \rightarrow \mathbb{R}$, which is the unique solution of the equation

$$
\pi_{1} f\left(x, g\left(x, x^{\prime}\right)\right)=x^{\prime} .
$$

It follows from (9.2) that $g \in C^{1}$. Let

$$
g^{\prime}\left(x, x^{\prime}\right)=\pi_{2} f\left(x, g\left(x, x^{\prime}\right)\right) .
$$

Consider the 1 -form

$$
\omega=g^{\prime}\left(x, x^{\prime}\right) d x^{\prime}-g\left(x, x^{\prime}\right) d x .
$$

Since $I(f)$ holds, this form is closed, i.e. exact (in $\mathbb{R}^{2}$ ). Therefore, there exists $L \in C^{2}\left(\mathbb{R}^{2}, \mathbb{R}\right)$ such that $\omega=d L$. Equations (9.4)-(9.6) yield (L3), (9.2) yields (L2).

Proof of Proposition 2.1. Since Lemma 9.1 holds, it is enough to check that for $\forall f \in \mathscr{D}$,

$$
I(f) \wedge I I(f) \Rightarrow[I I I(f) \Leftrightarrow(\mathrm{L} 1) \text { for the generating function of Lemma 9.1]. }
$$

Let $I(f) \wedge I I(f)$ be fulfilled. Since Lemma 9.1 holds, there exists a $C^{2}$-function $L\left(x, x^{\prime}\right)$ for which (L2) and (L3) are fulfilled. Let $z \in 3$ be a curve passing through the points $\left(x_{0}, y_{0}\right)$ and $\left(x_{0}+1, y_{0}\right)$. From (L3) it follows that $-y d x+y^{\prime} d x^{\prime}$ $=d L\left(x, x^{\prime}\right)$, where $\left(x^{\prime}, y^{\prime}\right)=f(x, y)$. Integrating along the segment, connecting points $\left(x_{0}, y_{0}\right)$ and $\left(x_{0}+1, y_{0}\right)$, one gets

$$
-\int_{x_{0}}^{x_{0}+1} \psi(x) d x+\int_{x_{0}}^{x_{0}+1} \tilde{\psi}(x) d x=L\left(x_{0}+1, x_{0}^{\prime}+1\right)-L\left(x_{0}, x_{0}^{\prime}\right),
$$

where graph $\psi=z$, graph $\tilde{\psi}=\tilde{z}=f(\mathfrak{z})$. This identity proves the required equivalence.

Proof of Proposition 5.1. The variables in the following formulas are related as it is shown in the diagram

$$
\begin{aligned}
& (x, y) \stackrel{f}{\mapsto}\left(x^{\prime}, y^{\prime}\right) \\
& g \downarrow \quad g \mp \\
& (\tilde{x}, \tilde{y}) \stackrel{\tilde{f}}{\mapsto}\left(\tilde{x}^{\prime}, \tilde{y}^{\prime}\right) .
\end{aligned}
$$

As $\tilde{f}=g \circ f \circ g^{-1}$, then

$$
d \tilde{f}(\tilde{x}, \tilde{y})=d g\left(x^{\prime}, y^{\prime}\right) \cdot d f(x, y) \cdot[d g(x, y)]^{-1}
$$

[see the notations in (2.1) and (9.9)].

The estimate (D3) for $\tilde{f}$ follows from the inequality $\|d \tilde{f}\| \leqq 8\|d g\|^{2}\|d f\|$, which in its turn follows from (9.10), (2.1)-(2.2). We introduce the following notations: $L\left(x, x^{\prime}\right)=L_{f}\left(x, x^{\prime}\right), S(x, \tilde{x})=L_{g}(x, \tilde{x}), \tilde{L}\left(\tilde{x}, \tilde{x}^{\prime}\right)$ is the generating function for $\tilde{f}$, which exists since Lemma 9.1 holds. According to the definition of generating functions we have

$$
\begin{aligned}
d S(x, \tilde{x}) & =-y d x+\tilde{y} d \tilde{x}, \\
d S\left(x^{\prime}, \tilde{x}^{\prime}\right) & =-y^{\prime} d x^{\prime}+\tilde{y}^{\prime} d \tilde{x}^{\prime}, \\
d L\left(x, x^{\prime}\right) & =-y d x+y^{\prime} d x^{\prime}, \\
d \tilde{L}\left(\tilde{x}, \tilde{x}^{\prime}\right) & =-\tilde{y} d \tilde{x}+\tilde{y}^{\prime} d \tilde{x}^{\prime} .
\end{aligned}
$$


Hence it follows that

$$
\begin{aligned}
& d\left[L\left(x, x^{\prime}\right)-\tilde{L}\left(\tilde{x}, \tilde{x}^{\prime}\right)\right]=d\left[S(x, \tilde{x})-S\left(x^{\prime}, \tilde{x}^{\prime}\right)\right], \\
& L\left(x, x^{\prime}\right)-\tilde{L}\left(\tilde{x}, \tilde{x}^{\prime}\right)=S(x, \tilde{x})-S\left(x^{\prime}, \tilde{x}^{\prime}\right)+\text { const } .
\end{aligned}
$$

As the functions $L$ and $S$ possess the property (L1), then from (9.13) it follows that the same is true for $\tilde{L}$. From Proposition 2.1 and (9.7) it follows that $\tilde{f} \in \mathscr{E}$. Let us reproduce (9.13) expressing all the terms as the functions of $(x, y)$ [see diagram (9.9)]. Let

$$
\begin{gathered}
F_{1}(x, y)=L\left(x, \pi_{1} f(x, y)\right), \quad F_{2}(x, y)=\tilde{L}\left(\pi_{1} g(x, y), \pi_{1} \tilde{f} \circ g(x, y)\right), \\
F_{3}(\tilde{x}, \tilde{y})=\tilde{L}\left(\tilde{x}, \pi_{1} \tilde{f}(\tilde{x}, \tilde{y})\right), \quad F_{4}(x, y)=S\left(x, \pi_{1} g(x, y)\right), \\
F_{5}(x, y)=S\left(\pi_{1} f(x, y), \pi_{1} \tilde{f} \circ g(x, y)\right) .
\end{gathered}
$$

According to the definition,

$$
F_{2}=g^{*} F_{3}, \quad F_{5}=f^{*} F_{4} .
$$

Calculating the value of $\mu \in \mathfrak{M}_{f}$ on the left and right of (9.13), we have

$$
P_{f}(\mu)-P_{\widetilde{f}} g_{*}(\mu)=\mu\left(F_{4}\right) \dot{-} f_{*} \mu\left(F_{4}\right)+\text { const } .
$$

The terms containing $\mu$ in the right part of $(9.15)$ cancel out due to the invariance of $\mu$ with respect to $f$.

\section{Proof of Proposition 6.1}

We use formula (9.10) substituting for $d g(x, y)$ the explicitly calculated matrix. Let $d f(x, y)=\left\{f_{i k}(x, y)\right\}_{i, k=1}^{2}$. Then in terms of the diagram (9.9)

$$
\begin{aligned}
\frac{\partial \pi_{1} \tilde{f}(\tilde{x}, \tilde{y})}{\partial \tilde{y}}= & f_{12}(x, y)-\varepsilon\left[f_{11}(x, y)+f_{12}(x, y)\left(\psi^{\prime}\left(x^{\prime}\right)+\psi^{\prime}(x)\right)-f_{22}(x, y)\right] \\
& +\varepsilon^{2}\left[\psi^{\prime}\left(x^{\prime}\right) f_{11}(x, y)-\psi^{\prime}(x) f_{22}(x, y)+\psi^{\prime}\left(x^{\prime}\right) \psi^{\prime}(x) f_{12}(x, y)-f_{21}(x, y)\right] .
\end{aligned}
$$

Due to (D3) the coefficients at $\varepsilon$ and $\varepsilon^{2}$ in the right part of (10.1) are uniformly bounded. Since $I I(f)$ holds, $\left|f_{12}(x, y)\right| \geqq c_{0}>0$. If $\varepsilon$ is sufficiently small, the right part of (10.1) is separated from zero.

\section{Proof of Remark 4.2}

The map $g(x, y)=(\tilde{x}, \tilde{y})$, where $\tilde{x}=x, \tilde{y}=y-\alpha$ transforms the curve $z$ invariant with respect to $f$ into zero curve $\tilde{z}$ invariant with respect to $\tilde{f}=g \circ f \circ g^{-1}$. It is easy to see that $\tilde{f} \in \mathscr{E}$, and $L_{\tilde{f}}\left(\tilde{x}, \tilde{x}^{\prime}\right)=L_{f}\left(\tilde{x}, \tilde{x}^{\prime}\right)+\alpha\left(\tilde{x}-\tilde{x}^{\prime}\right)$, and

$$
P_{\tilde{f}} g_{*}(\mu)=P_{f}^{(\alpha)}(\mu) \stackrel{\text { def }}{=} \int\left(L_{f}\left(x, x^{\prime}\right)+\alpha\left(x-x^{\prime}\right)\right) d \mu .
$$

Since $g_{*}$ maps measures $\mu \in \mathfrak{M}_{f}$ with $\operatorname{supp} \mu \mathcal{C}_{\mathfrak{z}}$ into measures $\tilde{\mu} \in \mathfrak{M}_{\tilde{f}}$ with $\operatorname{supp} \tilde{\mu} \subset \tilde{z}$, and is a bijection, then Remark 4.2 follows from Theorem A and formula (11.1). 
Acknowledgements. The authors learned of the problem in question from Ya. G. Sinai, who proposed that they investigate it. They thank Professor Sinai for this proposal and for continuing help during all the stages of the work.

\section{References}

1. Sinai, Ya.G.: Commensurate-incommensurate phase transitions in one-dimensional chains. J. Stat. Phys. 29, 401-425 (1982)

2. Sinai, Ya.G.: "Commensurability-noncommensurability" transition in one-dimensional chains. Zh. Eksp. Teor. Fiz. 83, 1223-1231 (1982) (Russian)

3. Frenkel, Y.I., Kontorova, T.A.: On the theory of the plastic deformation and twinning. Zh. Eksp. Teor. Fiz. 8, 89-95 (1938) (Russian)

4. Chirikov, B.V.: A universal instability of many-dimensional oscillator systems. Phys. Rep. 52: 5, 263-379 (1979)

5. Moser, J.: On invariant curves of area-preserving mappings of an annulus. Nachr. Akad. Wiss. Göttingen, Math.-Phys. K1. IIa, 1-20 (1962)

6. Katok, A.: Periodic and quasiperiodic orbits for twist maps. To appear in Springer Lecture Notes in Physics, 1983, Proc. VII Sitges Conference on Dynamical Systems and Chaos

7. Percival, I.C.: Variational principles for invariant tori and cantori. In: Nonlinear dynamics and the beam-beam interaction. AIP Conference Proceedings No. 57, pp. 302-310. New York: American Institute of Physics 1979

8. Mather, J.N.: Existence of quasiperiodic orbits for twist homeomorphisms of the annulus. Topology 21, 457-467 (1982)

9. Zaslavski, A.Ya.: Note on phase diagram in Frenkel-Kontorova model. Mat. Theor. Phys. 57, 459-464 (1983) (Russian)

10. Zehnder, E.: Generalized implicit function theorems with applications to some small division problems. I, II. I. Commun. Pure Appl. Math. 28, 91-141 (1975); II. Commun. Pure Appl. Math. 29, 49-113 (1976)

11. Hamilton, R.S.: The inverse function theorem of Nash and Moser. Bull. Am. Math. Soc. 7, 65-222 (1982)

12. Bak, Per: Commensurate phases, incommensurate phases, and the devil's staircase. Rep. Prog. Phys. 45, 587-629 (1982)

13. Aubry, S.: The devil's staircase transformation in incommensurate lattices. In: The Riemann problem, complete integrability, and arithmetic application. Lecture Notes in Mathematics, Vol. 925, pp. 221-245. Berlin, Heidelberg, New York: Springer 1982

14. Aubry, S., Le Daeron, P.Y.: The discrete Frenkel-Kontorova model and its extensions. Physica 8 D, 381-422 (1983)

Communicated by Ya. G. Sinai

Received January 10, 1984 